\title{
Glutamine: essential for immune nutrition in the critically ill
}

\author{
Francis J. Andrews and Richard D. Griffiths* \\ Intensive Care Research Group, Department of Medicine, Duncan Building, UCD, \\ Daulby Street, University of Liverpool, L69 3GA, UK
}

\begin{abstract}
Critically ill patients on intensive care units are at an increased risk of sepsis, which is a major cause of mortality in these patients. Recent evidence suggests that impairment of the functioning of the immune system contributes to the development of sepsis in such patients. In particular, monocytes show reduced expression of HLA-DR antigen, associated with impaired antigen presenting capability and decreased phagocytic activity; lymphocytes show decreased proliferation in response to mitogens and T-helper cells show a shift in the Th1/Th2 ratio consistent with impaired immunity. The amino acid glutamine becomes conditionally essential in the critically ill, yet such patients frequently have a marked deficiency of glutamine; the reasons for this are still unclear. Glutamine is required by the cells of the immune system both as a primary fuel and as a carbon and nitrogen donor for nucleotide precursor synthesis. In vivo studies have demonstrated that glutamine is essential for optimal immune cell functioning for monocytes, lymphocytes and neutrophils. A number of trials of patients fed by the enteral or parenteral route have shown improved infectious morbidity when supplemented with glutamine. However, the exact mechanism of glutamine action in these patients remains to be determined.
\end{abstract}

Glutamine: Immune system: Critical illness: Sepsis

\section{Introduction}

Following critical illness, sepsis remains a major cause of morbidity and mortality. Previously, the pathogenesis of sepsis and the systemic inflammatory syndrome (SIRS) was explained in terms of persistent, uncontrolled inflammation. However it is becoming increasingly apparent that counter regulatory mechanisms exist and this has been called the compensatory anti-inflammatory response syndrome (CARS). The relative magnitude of these responses determines the patient's response to a critical illness (Bone, 1996). Evidence is now accumulating to suggest that critically ill patients may be predisposed to infectious complications because of impaired function of the immune system resulting from CARS predominance, and this impairment may occur very early on in the illness. It is now established that specific nutritional supplementation can influence the immune response, a concept known as immunonutrition and much of this work has centred on the provision of the amino acid glutamine. Glutamine is the most abundant amino acid in the body and has the highest plasma concentration (typically $0.6 \mathrm{mmol} / \mathrm{l}$ ) of all the amino acids. It comprises about half the total free amino acid pool in skeletal muscle and under normal conditions is regarded as a non-essential amino acid as it can be produced in sufficient amounts to meet the body's needs (van Acker et al. 1999). Glutamine provides a source of energy through its partial oxidation in a process known as glutaminolysis (McKeehan, 1982) and also provides carbon and nitrogen for precursors of nucleotide synthesis; it is also a precursor of intracellular glutathione, hepatic glucose and urinary ammonia. During times of catabolic stress such as critical illness however, it becomes conditionally essential as a fuel for the cells of the immune system and the gastrointestinal system (Lacey \& Wilmore, 1990; Wernerman \& Hammarqvist, 1999). Deficiencies of glutamine supply seen in critical illness states are associated with impairment of the immune system and increased susceptibility to infection.

\section{Glutamine deficiency in the critically ill}

During critical illness, glutamine has been considered to be a 'conditionally essential' amino acid, with an increase in uptake in the kidneys, immune cells and intestinal mucosa. However, this increased demand is not met in severe illness, and such patients show significantly decreased plasma levels of glutamine. Plasma glutamine has been shown to be significantly decreased in patients with burns (Stinnett et al.

Abbreviations: BCG, Bacillus Calmette-Guerin; CARS, compensatory anti-inflammatory response syndrome; EN, enteral nutrition; GSH, glutathione; HSP, heat shock proteins; IFN $\gamma$, interferon gamma; IL, interleukin; LPS, lipopolysaccharide; PHA, phytohaemagglutinin; ROI, reactive oxygen intermediates; ROS, reactive oxygen species; SIRS, systemic inflammatory syndrome; Th, T-helper; TPN, total parenteral nutrition.

* Corresponding author: Dr R. D. Griffiths, fax +44 (0)151 706 5802, email rdg @liverpool.ac.uk 
1982; Parry-Billings et al. 1990), major trauma (Askanazi et al. 1980) and in a heterogeneous cohort of critically ill patients (Griffiths et al. 1997). Skeletal muscle is the main source of glutamine and there is a dramatic decline in muscle free glutamine levels in critical illness (Askanazi et al. 1980). It has been demonstrated by Mittendorfer et al. (1999) that the depletion of intramuscular glutamine in critically ill patients with burn injuries is not due to synthesis impairment or decreased appearance from protein breakdown but rather due to accelerated export from muscle cells. However there is evidence that the net intramuscular de novo synthesis of glutamine (the balance between glutamine synthesis and degradation) may be limited by a reduced availability of intramuscular glutamate as a precursor (Biolo et al. 2000). Increased transamination of glutamate to form alanine occurs and it is speculated that this is linked to the need to clear excess pyruvate. Addition of glutamine to total parenteral nutrition after abdominal surgery is associated with a significantly smaller fall in intracellular muscle glutamine than in control patients who received TPN (Hammarqvist et al. 1989).

\section{Impaired immune cell function in the critically ill}

\section{Monocytes and macrophages}

Monocytes are precursor cells for tissue macrophages and both are of central importance in the initiation, development and outcome of the immune response. Expression of class II major histocompatability complex antigens (HLA-DR and related antigens) is required for monocytes to present an antigen to lymphocytes and act as accessory cells. HLA-DR is also a marker of monocyte activation and thus the ability to phagocytose opsonized organisms. The monocytes of patients with septic shock have significantly less HLA-DR expression compared with those from normal subjects (Lin et al. 1993). It has been accepted until recently that patients with sepsis show a biphasic immunological pattern in which the early hyperinflammatory phase is counterbalanced by an anti-inflammatory response which may lead to a later hypoinflammatory state, characterised by monocyte deactivation (Kox et al. 2000). An important concept however is that many critically ill patients following trauma or major surgery have reduced HLA-DR expression on monocytes very early during the course of their illness (Wakefield et al. 1993; Ditschkowski et al. 1999; Giannoudis et al. 1999). Recently, immunoparalysis of monocytes has also been described for other groups of intensive care patients (Peters et al. 1999). Hershman et al. 1990 have shown that the downregulation of HLA-DR correlates with clinical outcome and sepsis in such patients. The reason for monocyte deactivation in CARS is not fully understood but there is increasing evidence that interleukin 10 (IL-10) plays a central role. Giannoudis et al. (2000) have demonstrated that immediate IL-10 expression following trauma regulates HLA-DR expression and is associated with sepsis development. Recovery of the monocyte function of patients destined to develop sepsis was markedly delayed compared with control subjects in this study.

\section{Lymphocytes}

In experimental models and in critically ill patients, various deficiencies of lymphocyte function have been described. These include depletion in total circulating lymphocyte numbers and particularly CD4+ lymphocytes, and a lowered proliferative response to mitogenic stimuli (Levy et al. 1984; Lin et al. 1993). CD4+ cells can differentiate into two types of effector cells and the pattern of circulating cytokines in critical illness determines CD4 Th (T-helper) lymphocyte subset predominance (Mossmann \& Coffman, 1989). Th1 cells produce IL-2 and interferon gamma (IFN $\gamma$ ) favouring cell mediated immunity whereas Th2 cells produce IL-4, IL-5, IL-6, IL-10h or IL-13, favouring humoral immunity. The Th2 antibody mediated immune response predominates in those patients with sepsis and it has been suggested that this may be due to markedly increased circulating concentrations of IL-10 (Sherry et al. 1996). A study by O'Sullivan et al. (1995) showed that patients with trauma and burns had increased levels of IL-4 and IL-10, suggesting a predominantly Th2 response, together with decreased levels of IL-12, a Th1-promoting cytokine. Ferguson et al. (1999) showed that the median ratio of Th1/Th2 ratio was 0.46 in patients with sepsis, and significantly lower than 2.5 in non-septic control patients. The decrease in the $\mathrm{Th} 1 / \mathrm{Th} 2$ ratio and consequent Th2 cytokine pattern dominance is associated with decreased resistance to infection (Neidhardt et al. 1997).

\section{Neutrophils}

Polymorphonuclear neutrophils have both regulatory and phagocytic functions. These cells synthesise and export cytokines and inflammatory mediators and also operate in the first line of defence against invading organisms. Excessive activation of neutrophils and consequent free radical release leads to organ dysfunction via tissue and endothelial cell damage (Fujishima \& Aikawa, 1995). However there is also evidence for neutrophil dysfunction in various forms during critical illness. Expression of the surface marker CD11b on neutrophils is decreased in patients who die of sepsis compared with controls (Muller Kobold et al. 2000), up-regulated CD11b being regarded as a marker of neutrophil activation. Release of the proinflammatory cytokine IL-8 from neutrophils in septic patients is significantly reduced when stimulated in vitro with lipopolysaccharide (LPS), when compared with neutrophils from healthy volunteers (Marie et al. 1998). Superoxide production by neutrophils has been shown to be reduced in critically ill patients with sepsis (Tschaikowsky et al. 1993). Simms \& D’Amico (1997) have also shown that the development of nosocomial infection on a surgical intensive care unit is associated with auto-oxidative injury to neutrophils (measured as a reduction in opsonic receptor expression induced by neutrophils themselves). Despite these observations, the exact prevalence of neutrophil dysfunction in critical illness is less well defined than for monocytes and lymphocytes. 


\section{Glutamine and the immune system}

\section{Dependence of immune cells on glutamine}

A striking feature of the cells of the immune system is that despite their differing function and cell biology, all are dependent on glutamine in a similar manner (Newsholme et al. 1999). All of these cells show high rates of glutamine consumption in culture; most of the glutamine is not fully oxidised (for energy) but converted to glutamate, lactate and aspartate. The high rates of glutamine utilisation are in excess of the needs for energy and nucleotide precursors and it has been hypothesised that the high rate of glutaminolysis provides for precision in regulating changes in the rate of synthesis of nucleotides (Newsholme et al. 1985). In addition, optimal phagocytic and secretory activity of immune cells may be dependent on adequate glutamine.

\section{Monocyte and macrophage function}

Monocytes and macrophages play an important role in the innate immune system response as a non-specific, first line defence and they are able to ingest and eliminate infectious agents. Macrophages are terminally differentiated cells derived from circulating blood monocytes but are highly active and characterised by high rates of protein secretion, however they are unable to synthesise glutamine themselves. Very high rates of glutamine utilisation occur in macrophages as shown by Newsholme et al. (1987). Studies by Parry-Billings et al. (1990) with mouse peritoneal macrophages showed that rates of phagocytosis declined at all glutamine concentrations in vitro below $0.6 \mathrm{mmol} / \mathrm{l}$ and that human lymphocyte proliferation was similarly reduced with decreasing glutamine concentrations. Wallace \& Keast (1992) further examined the role of glutamine in adequate monocyte function using thioglycollate-elicited peritoneal macrophages from mice. A decrease in culture medium glutamine from $0.5 \mathrm{mmol} / 1$ to $0.125 \mathrm{mmol} / 1$ was accompanied by a $25 \%$ decrease in RNA synthesis as measured by ${ }^{3} \mathrm{H}$-uridine incorporation, demonstrating the importance of adequate glutamine for nucleotide synthesis. IL-1 secretion from macrophages was also dependent on the presence of glutamine in the media, but only $0.03 \mathrm{mmol} / 1$ was required to restore sufficient IL-1 secretion. Blood monocyte-derived macrophage antigen expression and function in healthy volunteers is influenced in vitro by glutamine (Spittler et al. 1995). Monocyte culture in glutamine concentration of less than $0.6 \mathrm{mmol} / \mathrm{l}$ was associated with a significant decrease in cellular ATP levels and lowering the glutamine concentration from $2 \mathrm{mmol} / \mathrm{l}$ to $0.2 \mathrm{mmol} / \mathrm{l}$ reduced monocyte HLA-DR expression by $40 \%$ and decreased tetanus toxoid-induced antigen presentation.

\section{Lymphocyte function}

Glutamine acts as both a precursor of nucleotide synthesis and a primary fuel for lymphocytes, and high rates of utilisation occur even in resting lymphocytes (Ardawi, 1988). Chang et al. (1999a) have demonstrated that glutamine supplementation significantly enhanced phytohaemagglutinin (PHA)-stimulated lymphocyte proliferation; the production of both intracellular Reactive
Oxygen Species (ROS) and glutathione (GSH) were also enhanced following glutamine supplementation. O'Riordain et al. (1994) showed that T-cell DNA synthesis was increased in post-operative patients who received glutamine-supplemented total parenteral nutrition (TPN), but in this study, other lymphocyte functions and markers were not examined. Studies by Chang et al. (1999b) have shown that the addition of glutamine to lymphocytes in vitro in glutamine free media stimulated with live attenuated bacillus Calmette-Guerin (BCG) favours a Th1 response shown by the production of IFN $\gamma$. Further evidence that glutamine is important in facilitating the IL-2 response has come from studies of mice fed with glutamine-supplemented diets (Kew et al. 1999). Mice receiving supplemental glutamine showed significantly greater IL-2 production from concanavalin-stimulated lymphocytes compared with mice fed control diets. There was no significant increase in IL-4 production in cells from mice supplemented with glutamine.

\section{Neutrophil function}

The importance of glutamine for optimal neutrophil functioning is less well defined compared with what is known about the requirements for optimal function of lymphocytes and macrophages. Neutrophils play a pivotal role in host defence by phagocytosing and destroying invading bacteria. Although the energy substrate for neutrophils is glucose it is suggested by Vlessis et al. (1995) that neutrophils are able to utilise glutamine when glucose is restricted. A study of the effect of glutamine on neutrophil function in neutrophils from paediatric burn patients by Ogle et al. (1994) showed evidence of improved bactericidal function following glutamine supplementation in vitro. At glutamine concentrations of $1 \mathrm{mmol} / \mathrm{l}$ and above, bactericidal function was significantly enhanced when the killing of Staphylococcus aureus (S. aureus) was examined. However, no net uptake of glutamine by the cells could be detected. This paper also claimed that glutamine did not affect phagocytic activity or $\mathrm{C} 3 \mathrm{~b}$ receptors, but no conclusion can be drawn because of the very small sample size of patients used for this aspect of the study. Pithon Curi et al. (1997) have shown that rat neutrophils grown in isolated culture utilise glutamine at a higher rate than glucose. Furukawa et al. (1997) investigated the role of glutamine in the killing of Escherichia coli (E. coli) by neutrophils from postoperative patients. Neutrophils were cultured with opsonized $E$. coli in media supplemented with different concentrations of glutamine. The number of viable E. coli decreased by $26 \%$ when media was supplemented with $1 \mathrm{mmol} / \mathrm{l}$ glutamine compared with media supplemented with $0.5 \mathrm{mmol} / \mathrm{l}$ glutamine. However, different concentrations of in vitro glutamine supplementation caused no significant changes in the number of viable $E$. coli when cultured with neutrophils from healthy volunteers. The plasma concentrations of glutamine were significantly lower in patients than in controls.

A further study by Furukawa et al. (2000) examined the role of glutamine in the production of reactive oxygen intermediates (ROI) and phagocytosis in vitro by neutrophils from postoperative patients. These patients were found 
to have significantly decreased serum glutamine levels. Using a washed whole blood culture technique, they found that neutrophil ROI production and phagocytosis of fluorescent beads was significantly greater in the presence of $2 \mathrm{mmol} / \mathrm{l}$ glutamine than with lower or no glutamine supplementation. This study could be criticised on the grounds that no glucose at all was available to neutrophils once in culture, unlike the in vivo situation. A recent finding of interest is the effect of decreased plasma glutamine on the expression of heat shock proteins (HSP) in neutrophils. The heat shock response in inflammatory cells is supposed to provide self-protection of immune cells against ROI. Weingartmann et al. (1999) showed that compared to neutrophils from healthy volunteers, neutrophils in patients with polytrauma showed no HSP70 expression, in association with decreased plasma levels of glutamine. In lymphocytes of both patients and controls however, HSP70 expression was conserved.

\section{Effects of glutamine supplementation in the critically ill}

A number of randomised, double blind trials have demonstrated the efficacy of glutamine in reducing the infectious morbidity of patients with critical illness. Most of these studies have concentrated on glutamine supplementation to TPN, but some recent studies have also looked at glutamine-supplemented enteral nutrition (EN). Ziegler et al. (1992) demonstrated that the addition of glutamine to TPN initiated early following bone marrow transplantation reduced infection rates and microbial colonisation, associated with a decrease in the length of hospital stay. Griffiths et al. (1997) reported a significantly increased 6-month survival rate in patients who received glutaminesupplemented TPN compared with conventional TPN. Over three-quarters of the patients in this study were suffering from major sepsis on admission. Subsequent analysis showed that the excess mortality in the control patients was described by those fed for more than 5 days, who had progressively more and repeated acquired intensive infections yet encountered the same risk with a similar duration of parenteral nutrition and stay on ICU. Consistent with a possible cell mediated impaired T-lymphocyte function, control patients not given glutamine had more fungal infections and higher mortality (Griffiths et al. 2000). Following major abdominal surgery, Morlion et al. (1998) randomised patients to TPN with or without glutamine supplementation. They showed that following surgery, by day 3 the total lymphocyte count remained at normal levels in the patients receiving glutamine supplementation but declined in the control group. In addition, neutrophil function as assessed by the generation of cysteinylleukotrienes (potent lipid mediators containing all or part of the GSH molecule) was only maintained in those patients receiving glutamine.

Trials of enteral supplementation of glutamine have also been performed. Neu et al. (1997) studied enteral glutamine supplementation in very low birth weight infants. The difference in sepsis between the two groups was significant when birth weight was taken into account using a logistic regression analysis, with sepsis rates of $10 \%$ for glutamine-supplemented infants and $30 \%$ for control infants. Houdijk et al. (1998) conducted a randomised trial of glutamine-enriched EN on patients with multipletrauma, using infectious morbidity during the first 15 days as a primary endpoint. Those patients randomised to glutamine showed a significant decrease in the incidence of pneumonia, bacteraemia and sepsis, and significantly higher levels of plasma glutamine than in control patients. Measurement of the soluble TNF (Tumour Necrosis Factor) receptors p55 and p75 as markers of a systemic inflammatory response demonstrated that patients with glutamine supplementation had a lower systemic inflammatory response than in control patients. However it is notable that despite the placing of nasoduodenal tubes for enteral feeding, maximum plasma glutamine levels were not achieved until day 5 even in the glutamine supplementation group. The same group also analysed endocrine and metabolic mediators from this study and found that the reduction in infectious morbidity could not be explained by modulation of the humoral stress response (Houdijk et al. 1999).

\section{Conclusion}

Recent evidence has accumulated to suggest that some critically ill patients may be at increased risk of infection because of impaired immune function early in the course of their illness. Studies have demonstrated that there is good evidence for monocyte deactivation and increasing evidence for lymphocyte and neutrophil dysfunction. Many of these functions have been shown to be dependent on the presence of glutamine, which is often deficient in supply in the critically ill. It would therefore seem logical that consideration should be given to giving glutamine very early on in the course of a critical illness to firstly remedy any immune system dysfunction and secondarily to prevent any further deactivation of immune cells. Despite this impressive evidence for glutamine supplementation in the critically ill, none of these trials have investigated whether it is a single or combination of mechanisms by which glutamine has exerted its effect, and further studies are needed to clarify these mechanisms.

\section{References}

Ardawi MSM (1988) Glutamine and glucose metabolism in peripheral blood lymphocytes. Metabolism 37, 99-103.

Askanazi J, Carpentier YA, Michelsen CB, Elwyn DH, Furst P, Kantrowitz LR, Gump FE \& Kinney JM (1980) Muscle and plasma amino acids following injury. Annals of Surgery 192, $78-85$.

Biolo G, Fleming RYD, Maggi SP, Nguyen TT, Herndon DN \& Wolfe RR (2000) Inhibition of muscle glutamine formation in hypercatabolic patients. Clinical Science 99, 189-194.

Bone RC (1996) Sir Isaac Newton, Sepsis, SIRS, and CARS. Critical Care Medicine 24, 1125-1127.

Chang WK, Yang KD \& Shaio MF (1999a) Effect of glutamine on Th1 and Th2 cytokine responses of human peripheral blood mononuclear cells. Clinical Immunology 93, 294-301.

Chang WK, Yang KD \& Shaio MF (1999b) Lymphocyte proliferation modulated by glutamine: involved in the 
endogenous redox reaction. Clinical and Experimental Immunology 117, 482-488.

Ditschkowski M, Kreuzfelder E, Regmann V, Ferencik S, Majestschak M, Schmid EN, Obertacke U, Hirche H, Schade UF \& Grosse-Wide H (1999) HLA-DR expression and soluble HLA-DR levels in septic patients after trauma. Annals of Surgery 229, 246-254.

Ferguson NR, Galley HF \& Webster NR (1999) T-helper cell subset ratios in patients with severe sepsis. Intensive Care Medicine 25, 106-109.

Fujishima S \& Aikawa N (1995) Neutrophil-mediated tissue injury and its modulation. Intensive Care Medicine 21, 277-285.

Furukawa S, Saito H, Fukatsu K, Hashiguchi Y, Inaba T, Lin MT, Inoue T, Han I, Matsuda T \& Muto T (1997) Glutamineenhanced bacterial killing by neutrophils from postoperative patients. Nutrition 13, 863-869.

Furukawa S, Saito H, Inoue T, Matsuda T, Fukatsu K, Han I, Ikeda S \& Hidemura A (2000) Supplemental glutamine augments phagocytosis and reactive oxygen intermediate production by neutrophils and monocytes from postoperative patients in vitro. Nutrition 16, 323-329.

Giannoudis PV, Smith RM, Perry SL, Windsor AJ, Dickson RA \& Bellamy MB (2000) Immediate IL-10 expression following major orthopaedic trauma: relationship to anti-inflammatory response and subsequent development of sepsis. Intensive Care Medicine 26, 1076-1081.

Giannoudis PV, Smith RM, Windsor AC, Bellamy MC \& Gillou PJ (1999) Monocyte Human Leucocyte antigen-DR expression correlates with intrapulmonary shunting after major trauma. American Journal of Surgery 177, 455-459.

Griffiths RD, Allen KD \& Jones C (2000) Glutamine TPN and intensive care acquired infections. Clinical Nutrition 19, 42 (abstract 172).

Griffiths RD, Jones C \& Palmer TEA (1997) Six-month outcome of critically ill patients given glutamine-supplemented parenteral nutrition. Nutrition 4, 296-302.

Hammarqvist F, Wernerman J, Custom A, Von der Deacon A \& Vines E (1989) Addition of glutamine to total parenteral nutrition after elective abdominal surgery spares free glutamine in muscle, counteracts the fall in muscle protein synthesis, and improves nitrogen balance. Annals of Surgery 209, 455-461.

Hershman MJ, Cheadle WG, Wellhausen SR, Davidson PF \& Polk HC (1990) Monocyte HLA-DR antigen expression characterises clinical outcome in the trauma patient. British Journal of Surgery 77, 204-207.

Houdijk APJ, Nijveldt RJ \& van Leeuwen PAM (1999) Glutamineenriched enteral feeding in trauma patients: reduced infectious morbidity is not related to changes in endocrine and metabolic responses. Journal of Parenteral and Enteral Nutrition 23, S52-S58.

Houdijk APJ, Rijnsburger ER, Jansen J, Wesdorp RIC, Weis JK, McCamish MA, Teerlink T, Meuwissen SG, Haarman Hj, Thijs LG \& van Leeuwen PA (1998) Randomised trial of glutamineenriched enteral nutrition on infectious morbidity in patients with multiple trauma. Lancet 352, 772-776.

Kew S, Wells S, Yaqoob P, Wallace FA, Miles EA \& Calder PC (1999) Dietary glutamine enhances murine T-lymphocyte responsiveness. Journal of Nutrition 129, 1524-1531.

Kox WJ, Volk T, Kox SN \& Volk HD (2000) Immunomodulatory therapies in sepsis. Intensive Care Medicine 26, S103-S110.

Lacey JM \& Wilmore DW (1990) Is glutamine a conditionally essential amino acid? Nutrition Reviews 48, 297-309.

Levy EM, Alharbi SA, Grindlinger G \& Black PH (1984) Changes in mitogen responsiveness lymphocyte subsets after traumatic injury: relation to development of sepsis. Clinical Immunology and Immunopathology 32, 224-233.

Lin RY, Astir ME, Saxon JC \& Rack EC (1993) Altered leucocyte immunophenotypes in septic shock. Studies of HLA-DR, CD11b, CD14 and IL-2R expression. Chest 104, 847-853.

Marie C, Muret J, Fitting C, Losser MR, Payen D \& Cavaillon JM (1998) Reduced ex vivo interleukin-8 production by neutrophils in septic and nonseptic systemic inflammatory response syndrome. Blood 91, 3439-3446.

McKeehan WL (1982) Glycolysis, glutaminolysis and cell proliferation. Cell Biology International Reports 6, 635-647.

Mittendorfer B, Gore DC, Herndon DN \& Wolfe RR (1999) Accelerated glutamine synthesis in critically ill patients cannot maintain normal intra muscular free glutamine concentration. Journal of Parenteral and Enteral Nutrition 23, 243-250.

Morlion BJ, Stehle P, Wachter P, Siedhoff HP, Köller M, König W, Fürst P \& Puchstein C (1998) Total parenteral nutrition with glutamine dipeptide after major abdominal surgery. Annals of Surgery 227, 302-308.

Mossmann TR \& Coffman RL (1989) TH1 and TH2 cells: different patterns of lymphokine secretion lead to different functional properties. Annual Review of Immunology 7, 145-173.

Muller Kobold AC, Tulleken JE, Zijlstra JG, Sluiter W, Hermans J, Kallenberg CG \& Cohen Tervaert JW (2000) Leucocyte activation in sepsis; correlations with disease state and mortality. Intensive Care Medicine 26, 883-892.

Neidhardt R, Keel M, Steckholzer U, Safret A, Ungethuem U, Tentz O \& Ertel W (1997) Relationship of interleukin-10 plasma levels to severity of injury and clinical outcome in injured patients. Journal of Trauma 42, 863-870.

Neu J, Roig JC, Meetze WH, Veerman M, Carter C, Millsaps M, Bowling D, Dallas MJ, Sleasman J, Knight T \& Auestad N (1997) Enteral glutamine supplementation for very low birthweight infants decreases morbidity. Journal of Pediatrics 131, 691-699.

Newsholme EA, Crabtree B \& Ardawi MSM (1985) The role of high rates of glycolysis and glutamine utilisation in rapidly dividing cells. Bioscience Reports 5, 393-400.

Newsholme EA, Crabtree B \& Ardawi MSM (1987) Rates of utilisation and fates of glucose, glutamine, pyruvate, fatty acids and ketone bodies by mouse macrophages. Biochemical Journal 242, 631-636.

Newsholme P, Curi R, Pithon Curi TC, Murphy CJ, Garcia C \& Pires de Melo M (1999) Glutamine metabolism by lymphocytes, macrophages and neutrophils: its importance in health and disease. Journal of Nutritional Biochemistry 10, 316-324.

Ogle CK, Ogle JD, Mao JX, Simon J, Noel JG, Li BG \& Alexander JW (1994) Effect of glutamine on phagocytosis and bacterial killing by normal and paediatric burn patient neutrophils. Journal of Parenteral and Enteral Nutrition 18, 128-133.

O'Riordain MG, Fearon KCH, Ross JA, Rogers P, Falconer JS, Bartolo DCC, Garden OJ \& Carter DC (1994) Glutaminesupplemented total parenteral nutrition enhances T-lymphocyte response in surgical patients undergoing colorectal resection. Annals of Surgery 220, 212-221.

O'Sullivan ST, Lederer JA, Horgan AF, Chin DH, Mannick JA \& Rodrick ML (1995) Major injury leads to predominance of the T-helper-2 lymphocyte phenotype and diminished interleukin12 production associated with decreased resistance to infection. Annals of Surgery 222, 482-490.

Parry-Billings M, Evans J, Calder P \& Newsholme EA (1990) Does glutamine contribute to immunosuppression after major burns? Lancet 336, 523-525.

Peters M, Petros A, Dixon G, Inwald D \& Klein N (1999) Acquired immunoparalysis in paediatric intensive care: prospective observational study. British Medical Journal 319, 609-610.

Pithon Curi TC, Pires de Melo M, De Azevedo R, Zorn TMT \& Curi R (1997) Glutamine utilization by rat neutrophils: Presence of phosphate-dependent glutaminase. American Journal of Physiology 273, C1124-C1129. 
Sherry RM, Cue JI, Goddard JK, Parramore JB \& DiPiro JT (1996) Interleukin-10 is associated with the development of sepsis in trauma patients. Journal of Trauma 40, 613-616.

Simms HH \& D'Amico R (1997) Posttraumatic auto-oxidative polymorphonuclear neutrophil receptor injury predicts the development of nosocomial infection. Archives of Surgery 132, $171-177$.

Spittler A, Winkler S, Gotzinger P, Oehler R, Willheim M, Tempfer C, Weigel G, Fugger R, Boltz-Nitulescu G \& Roth E (1995) Influence of glutamine on the phenotype and function of human monocytes. Blood 86, 1564-1569.

Stinnett JD, Alexander JW, Watanabe C, MacMillan BG, Fischer JE, Morris MJ, Trocki O, Miskell P, Edwards L \& James H (1982) Plasma and skeletal muscle aminoacids following severe burn injury in patients and experimental animals. Annals of Surgery 195, 75-89.

Tschaikowsky K, Sittl R, Braun GG, Hering W \& Rügheimer E (1993) Increased fMet-leu-phe receptor expression and altered superoxide of neutrophil granulocytes in septic and posttraumatic patients. Clinical Investigation 72, 18-25.

Van Acker BAC, von Meyenfeldt MF, van der Hulst RRWJ, Hulsewé KWE, Wagenmakers AJM, Deutz NEP, de Blaauw I, Dejong CHC, van Kreel BK \& Soeters PB (1999) Glutamine: the pivot of our nitrogen economy? Journal of Parenteral and Enteral Nutrition 23, S45-S48.

Vlessis AA, Goldman RK \& Trunkey DD (1995) New concepts in the pathophysiology of oxygen metabolism during sepsis. British Journal of Surgery 82, 870-876.

Wakefield CH, Carey PD, Foulds S, Monson JRT \& Gillou PJ (1993) Changes in major histocompatibility complex class II expression in monocytes and $\mathrm{T}$ cells of patients developing infection after surgery. British Journal of Surgery 77, 204-207.

Wallace C \& Keast D (1992) Glutamine and macrophage function. Metabolism 41, 1016-1020.

Weingartmann G, Oehler R, Derkits S, Oismüller C, Függer R \& Roth E (1999) HSP70 expression in granulocytes and lymphocytes of patients with polytrauma: comparison with plasma glutamine. Clinical Nutrition 18, 121-124.

Wernerman J \& Hammarqvist F (1999) Glutamine: a necessary nutrient for the intensive care patient. International Journal of Colorectal Disease 14, 137-142.

Ziegler TR, Young LS, Benfell K, Scheltinga M, Hortos K, Bye R, Morrow FD, Jacobs DO, Smith RJ, Antin JH \& Wilmore DW (1992) Clinical and metabolic efficacy of glutaminesupplemented parenteral nutrition after bone marrow transplantation. Annals of Internal Medicine 116, 821-828. 